\title{
Urgent and emergency surgery for secondary peritonitis during the COVID-19 outbreak: an unseen burden of a healthcare crisis
}

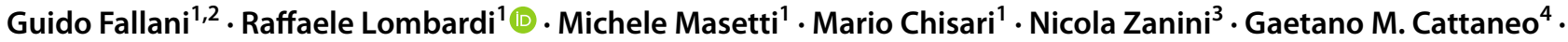 \\ Mauro Filosa ${ }^{4}$ - Federico Zanzi $i^{5}$. Enrico Guerra ${ }^{5}$. Stefano Bonilauri ${ }^{6}$. Luca Di Donato ${ }^{6}$. Gianluca Garulli ${ }^{3}$. \\ Andrea Lucchi ${ }^{7}$. Michele Grassia ${ }^{7}$. Giampaolo Ugolini ${ }^{8,2}$. Francesco Pasini ${ }^{8,2}$. Gaetano Vetrone ${ }^{9}$. Claudia Benini ${ }^{1}$. \\ Simone Nicosia ${ }^{1} \cdot$ Elio Jovine ${ }^{1}$
}

Received: 24 August 2020 / Accepted: 7 December 2020 / Published online: 4 January 2021

(c) Italian Society of Surgery (SIC) 2021

\begin{abstract}
The COVID-19 pandemic has raised concerns about the negative impact of the fear of contagion on people's willingness to seek medical care and the subsequent effects on patients' prognosis. To date, not much is known about the outcomes of acute surgical diseases in this scenario. The aim of this multicenter observational study is to explore the effects of COVID19 outbreak on the outcomes of patients who underwent surgery for peritonitis. Patients undergoing surgery for secondary peritonitis during the first COVID-19 surge in Italy (March 23-May 4, 2020-COVID period group) were compared with patients who underwent surgery during the same time interval of year 2019 (no-COVID period group). The primary endpoint was the development of postoperative complications. Logistic regression analysis was conducted to identify predictors of complications. Of the 332 patients studied, 149 were in the COVID period group and 183 were in the no-COVID period group. Patients in the COVID period group had an increased frequency of late presentations to the emergency departments (43\% vs. $31.1 \% ; P=0.026)$ and a higher rate of postoperative complications $(35.6 \%$ vs. $18 \% ; P<0.001)$. The same results were found in the subset analysis of patients with severe peritonitis at surgical exploration. The ASA score, severity of peritonitis, qSOFA score, diagnosis other than appendicitis, and COVID period resulted independent predictors of complications. During the COVID-19 pandemic patients with peritonitis had a higher rate of complicated postoperative courses, weighing on hospital costs and assistance efforts already pressured by the ongoing sanitary crisis.
\end{abstract}

Keywords Peritonitis · Emergency surgery $\cdot$ COVID-19

Guido Fallani and Raffaele Lombardi contributed equally to this work.

Raffaele Lombardi

raffaele.lombardi@ausl.bologna.it

1 Division of General and Emergency Surgery, Department of Specialistic Surgery, Ospedale Maggiore, IRCCS Azienda Ospedaliero-Universitaria Di Bologna, Largo Bartolo Nigrisoli, 2, 40133 Bologna, Italy

2 Department of Medical and Surgical Sciences (DIMEC), University of Bologna, Bologna, Italy

3 Division of Surgery, Ospedale "Infermi”, AUSL Romagna, Rimini, Italy

4 Division of General, Vascular and Thoracic Surgery, Ospedale "Guglielmo da Saliceto", AUSL Piacenza, Piacenza, Italy
5 Division of Surgery, Ospedale "Santa Maria Delle Croci", AUSL Romagna, Ravenna, Italy

6 Division of Surgery, Arcispedale "Santa Maria Nuova", AUSL Reggio Emilia, Reggio Emilia, Italy

7 Division of Surgery, Ospedale "Ceccarini”, AUSL Romagna, Riccione, Italy

8 Division of Surgery, Ospedale degli Infermi, AUSL Romagna,, Faenza, Italy

9 Division of Surgery, Ospedale "Santa Maria Della Scaletta", AUSL Imola, Imola, Italy 


\section{Introduction}

Since January 2020, the world has faced a global pandemic of coronavirus disease 2019 (COVID-19), an infectious disease caused by severe acute respiratory syndrome coronavirus 2 (SARS-CoV-2).

After the first Italian case was diagnosed on February 20 [1], Italy became the first country outside of Asia to suffer a major coronavirus emergency. As of November 2020, Italy is facing a new surge of contagions and remains one of the hardest-hit European countries [2], being Emilia-Romagna the Italian region with the secondhighest death rate [3].

On March 9, to contain the outbreak, the Italian government imposed a national quarantine with several restrictive measures (i.e., self-isolation, limitation of movements, and temporary closure of inessential businesses), which were partially relaxed on May 4. Such lockdown measures were unprecedented in the history of the Republic and represented a previously unforeseen, although necessary, suppression of constitutional civil rights.

This scenario, together with the broad media coverage of COVID-19-related deaths, resulted in social withdrawal behaviors above those required because of fear and anxiety about the perceived risk of infection. Such behaviorsunthinkable before the COVID-19 global spread-have no antecedents in Western medical literature but were accurately depicted in the description of the 1630 plague outbreak in Milan by Alessandro Manzoni, author of The Betrothed.

According to a Gallup poll conducted between March 28 and April 2 in the United States, $83 \%$ of respondents said they would be "very concerned" or "moderately concerned" about exposure to the coronavirus if they needed medical treatment at a doctor's office or hospital [4].

In the areas, most affected by the pandemic, these concerns dramatically influenced people's willingness to seek medical care, even to the point of avoiding acute care. Mantica et al. [5], studying the numbers of emergency department (ED) visits in two major referral hospitals in northern Italy concerning COVID-19 daily mortality from February 21 to April 16, found that the lowest numbers of ED visits corresponded with the highest numbers of deaths from COVID-19. These findings suggested that "the fear of what we can get might be greater than the fear of what we have" (p. 40). Many other studies in different disciplines have confirmed a significant reduction of ED visits during the COVID-19 outbreak [6-8], which raises concern about the effect of this behavior on patients' prognoses. To date, few cases of delayed treatment of acute abdomen during the coronavirus pandemic have been reported [9]. This study aimed to evaluate the effect of the COVID-19 outbreak on timing of access to ED and outcomes of patients undergoing urgent and emergency surgery for secondary peritonitis.

\section{Methods}

\section{Study population}

Consecutive patients referred from the ED to undergo urgent or emergency surgery for secondary localized or diffuse peritonitis during the COVID-19 lockdown period (March 23-May 4, 2020) in eight hospitals throughout the EmiliaRomagna region, Italy, were prospectively evaluated for enrollment. Exclusion criteria were diagnosis of acute cholecystitis, onset of peritonitis during hospitalization for other diseases, and positive result of oropharyngeal swab testing for SARS-CoV-2 or, before implementation of COVID-19 screening for surgical patients, perioperative symptoms/ radiologic findings suggestive of COVID-19. Patients with acute cholecystitis were excluded because management of this condition varied widely across hospitals.

The remaining patients constituted the final cohort (COVID period group), which was stratified according to the cause of peritonitis. The same criteria were used to retrospectively select consecutive patients who underwent surgery within the same 43-day time interval (March 23 through May 4) of 2019 as controls (no-COVID period group). The demographics, clinical characteristics, intraoperative findings, and postoperative outcomes of the two groups were compared.

The primary endpoint of the study was postoperative morbidity. The secondary endpoint was the timing of care, including diagnostic and treatment intervals.

\section{Data collection}

A database was created for the purpose of this study in a typical Excel (Microsoft Corporation-Redmond, WA, USA) spreadsheet. To ensure consistency in data entry, free-text entries were avoided as much as possible, and the admitted values for each relevant variable were restricted to a predefined cluster. The template of the database, together with a data dictionary, was then provided to each institution; any coding questions were referred to the principal investigator. Before the statistical tests were conducted, the master database was checked centrally for quality, and in cases of missing, unexpected, or ambiguous data, the local lead investigator was contacted for updating or clarification.

The data collected concerned: age, sex, comorbidities, body mass index (BMI), previous abdominal surgery, referral delay, white blood cell (WBC) count, percentage of polymorphonuclear neutrophils (\% PMN), C-reactive 
protein (CRP) levels, severity of organ dysfunction, diagnostic imaging type (ultrasonography [US], computed tomography [CT], or plain radiography], imaging findings, operation delay, patient's preoperative physical status, causes of peritonitis, severity of peritonitis, type of operation, operative approach (open vs. laparoscopic), conversion to open surgery, duration of surgery, postoperative complications, length of postoperative hospital stay (LOS), hospital readmissions, and postoperative mortality.

Due to the observational nature of the study, subjects were not contacted after index hospitalization discharge, and therefore data about clinical outcomes up to 30 days after surgery were extracted from the outpatient visit reports (for complications) and administrative databases (for mortality). Patients who developed complications within 30 days of surgery were monitored until recovery or death.

\section{Variable definitions and outcome measures}

Complications were defined as any deviation from the normal postoperative course that is not inherent to the procedure and that does not imply failure to cure [10]. For each patient, the postoperative complications were graded individually according to the Clavien-Dindo classification of surgical complications [10] and summarized with the Comprehensive Complication Index (CCI®) [11].

Referral delay was defined as the time from symptom onset to ED presentation. Operation delay was defined as the time from ED presentation to initial intervention. The total delay was the sum of the referral and operation delays.

Organ dysfunction was scored according to the Quick Sequential [Sepsis-related] Organ Failure Assessment (qSOFA) [12].

Disease severity was evaluated at surgical exploration and classified according to the American Association for the Surgery of Trauma (AAST) anatomic grading scales in emergency general surgery [13]. AAST grades IV (for appendicitis and perforated peptic ulcer only) and V (for all conditions) were defined as severe peritonitis.

To allow the individual risk stratification in association with concomitant diseases and physical status, the Charlson Age-Comorbidity Index (CACI) $[14,15]$ and the American Society of Anesthesiologists (ASA) classification [16] were used.

Previous abdominal surgery was defined as any prior open or laparoscopic procedure in which the peritoneal cavity was entered. Conversion to open surgery was defined as cessation of the laparoscopic approach and the use of a conventional laparotomy incision to complete the surgical procedure.

\section{Statistical analysis}

Values for categorical and ordinal variables were calculated as numbers and percentages; those for continuous variables were calculated as either means \pm standard deviations or medians and interquartile ranges (IQRs) depending on their distribution. Univariable analysis was conducted with the chi-square test or Fisher's exact test for categorical and ordinal variables, depending on the number of the patients, and with analysis of variance or the Mann-Whitney $U$ test for continuous variables, depending on their distribution. Variables with differences of $P<0.1$ were entered into a logistic regression model for multivariable analysis. Differences of $P<0.05$ were considered significant. All statistical analyses were conducted with SPSS version 23 (IBM Corporation-Armonk, NY, USA).

\section{Results}

\section{Study population characteristics}

Of the 332 subjects included in the study, 149 were in the COVID period group and 183 in the no-COVID period group. From each participating hospital data from a median of 40 patients (IQR: 26-51) were obtained. Overall, the most common reason for surgery was appendicitis $(n=238 ; 71.7 \%)$; the other reasons were acute diverticulitis $(n=51 ; 15.4 \%)$, perforated peptic ulcer $(n=30 ; 9 \%)$, and small bowel perforation $(n=13 ; 3.9 \%)$.

The demographics and preoperative characteristics of the patients by period of surgery are listed in Table 1 . Age, sex, BMI, comorbidities, previous abdominal surgery, and the ASA score were not significantly different between the two groups. Patients undergoing surgery during COVID-19 period had slightly longer referral delays than did those who underwent surgery before the COVID19 period [28 h (IQR: 12-48) vs. 24 h (IQR: 12-72); $P=0.004$ ] (Fig. 1); moreover, during COVID-19 period a higher rate of patients presented to the ED after 48 or more hours from symptoms onset ( $43 \%$ vs. $31.1 \%, P=0.026$ ). Operation delays were similar in the two groups. High qSOFA scores (1-3) were more common among the patients in the COVID period group than among those in the no-COVID period group ( $18.8 \%$ vs. $7.1 \% ; P=0.007)$, and the rate of CT performed in the ED was higher in the former group than in the latter group $(64.5 \%$ vs. $47 \%$; $P=0.005)$. Regarding the cause of peritonitis, the COVID period group, in comparison with the no-COVID period group, demonstrated less appendicitis ( $62.4 \%$ vs. $79.2 \%$; $P=0.001)$ and more perforated peptic ulcers $(12.8 \%$ vs. $6 \% ; P=0.033)$. 
Table 1 Demographics and preoperative characteristics by period of operation

\begin{tabular}{|c|c|c|c|}
\hline Variable & COVID period $(n=149)$ & $\begin{array}{l}\text { no-COVID period } \\
(n=183)\end{array}$ & $P$ value \\
\hline Age in years, median [IQR] & $49[26.5-70]$ & $44[24-61]$ & 0.223 \\
\hline Sex, $n(\%)$ & & & 0.065 \\
\hline Female & $55(36.9 \%)$ & $86(47 \%)$ & \\
\hline Male & $94(63.1 \%)$ & $97(53 \%)$ & \\
\hline $\mathrm{BMI}$ in $\mathrm{kg} / \mathrm{m}^{2}$, mean $\pm \mathrm{STD}$ & $25.3 \pm 2.1$ & $25.2 \pm 2.4$ & 0.675 \\
\hline $\mathrm{CACI}>3, n(\%)$ & $33(22.1 \%)$ & $32(17.5 \%)$ & 0.287 \\
\hline Previous abdominal surgery, $n(\%)$ & & & 0.474 \\
\hline No surgery & $115(77.2 \%)$ & $151(82.5 \%)$ & \\
\hline Laparoscopic surgery & $8(5.4 \%)$ & $7(3.8 \%)$ & \\
\hline Open surgery & $26(17.4 \%)$ & $25(13.7 \%)$ & \\
\hline WBC count in $10^{9} / \mathrm{L}$, median $[\mathrm{IQR}]$ & $13,5[10.7-17.8]$ & $13[9.9-16]$ & 0.177 \\
\hline $\mathrm{PMN}$ in $\%$, mean $\pm \mathrm{STD}$ & $81.3 \pm 11.2$ & $80.1 \pm 9.3$ & 0.299 \\
\hline $\mathrm{CRP}$ in $\mathrm{mg} / \mathrm{dL}$, median [IQR] & $12.6[3.1-27]$ & $11.4[2.4-31.2]$ & 0.827 \\
\hline qSOFA score, $n(\%)$ & & & 0.007 \\
\hline 0 & $119(81 \%)$ & $165(92.7 \%)$ & \\
\hline 1 & $21(14.3 \%)$ & $8(4.5 \%)$ & \\
\hline 2 & $7(4.8 \%)$ & $4(2.2 \%)$ & \\
\hline 3 & 0 & $1(0.6 \%)$ & \\
\hline Missing & $2(1.3 \%)$ & $5(2.7 \%)$ & \\
\hline Diagnostic imaging type, $n(\%)$ & & & 0.005 \\
\hline US & $47(31.5 \%)$ & $90(49.2 \%)$ & \\
\hline $\mathrm{CT}$ & $96(64.5 \%)$ & $86(47 \%)$ & \\
\hline Plain radiography & $6(4 \%)$ & $7(3.8 \%)$ & \\
\hline \multicolumn{4}{|l|}{ Imaging findings, $n(\%)$} \\
\hline Abscess & $38(25.5 \%)$ & $46(25.1 \%)$ & 0.939 \\
\hline Free fluid & $104(69.8 \%)$ & $100(54.6 \%)$ & 0.005 \\
\hline Free air & $40(26.8 \%)$ & $36(19.7 \%)$ & 0.122 \\
\hline Referral delay in hours, median [IQR] & $28[12-72]$ & $24[12-48]$ & 0.004 \\
\hline Referral delay $\geq 48$ h, $n(\%)$ & $64(43 \%)$ & $57(31.1 \%)$ & 0.026 \\
\hline Operation delay in hours, median [IQR] & $10[5-20]$ & $11[6-24]$ & 0.337 \\
\hline Total delay in hours, median [IQR] & $42.5[24-82.5]$ & $36[20-71]$ & 0.165 \\
\hline ASA score, $n(\%)$ & & & 0.272 \\
\hline 1 & $56(37.6 \%)$ & $77(42.1 \%)$ & \\
\hline 2 & $49(32.9 \%)$ & $64(35 \%)$ & \\
\hline 3 & $31(20.8 \%)$ & $35(19.1 \%)$ & \\
\hline 4 & $13(8.7 \%)$ & $7(3.8 \%)$ & \\
\hline \multicolumn{4}{|l|}{ Cause of peritonitis, $n(\%)$} \\
\hline Perforated peptic ulcer & $19(12.8 \%)$ & $11(6 \%)$ & $\mathbf{0 . 0 3 3}$ \\
\hline Small bowel perforation & $8(5.4 \%)$ & $5(2.7 \%)$ & 0.218 \\
\hline Appendicitis & $93(62.4 \%)$ & $145(79.2 \%)$ & 0.001 \\
\hline Acute diverticulitis & $29(19.5 \%)$ & $22(12.0 \%)$ & 0.061 \\
\hline
\end{tabular}

Significant $P$ values are in bold

\section{Intraoperative findings and postoperative outcomes}

As reported in Table 2, patients who underwent surgery during the COVID period, in comparison with those who underwent surgery before the COVID period, had a higher rate of severe peritonitis ( $67.8 \%$ vs. $55.2 \% ; P=0.019)$, and were more likely to have final open (i.e., primary open and laparoscopic converted) surgery (33.6\% vs. 20.8\%; $P=0.012$ ). The median operation time was $75 \mathrm{~min}$ (IQR: 50-123) for the COVID period group and $60 \mathrm{~min}$ (IQR: $40-85)$ for the no-COVID period group $(P<0.001)$. 


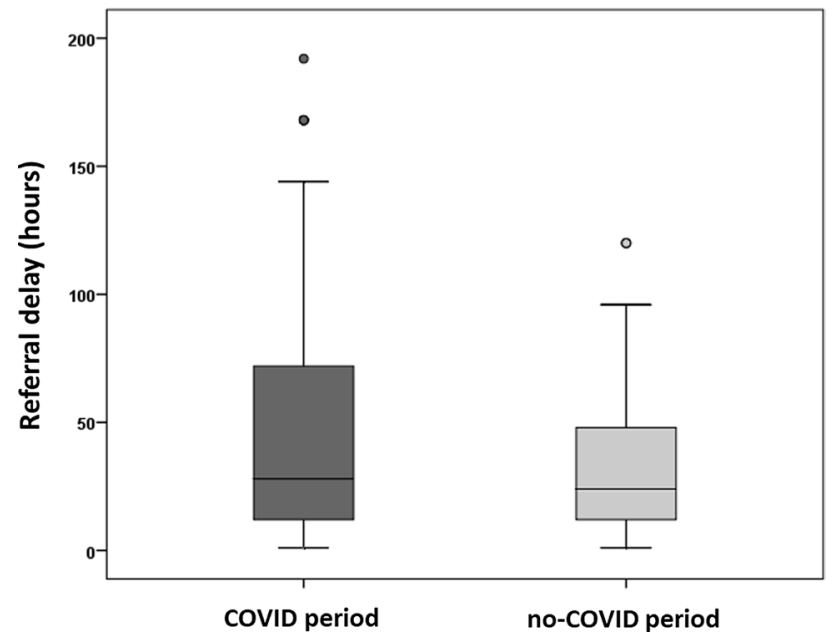

Fig. 1 Referral delay by period, boxplot

The unadjusted rate of overall postoperative complications was twofold higher among patients who underwent surgery during the COVID period than among those who underwent surgery before the COVID period (35.6\% vs. $18 \% ; P<0.001)$, as was the overall burden of postoperative morbidity measured with the $\mathrm{CCI} 囚$. No difference in major complications was detected between the two groups. In the postoperative period, four patients in the COVID period group developed symptomatic SARS-CoV-2 infection, two of whom required intensive care. Thirty days after surgery, all these patients were in stable or improved condition.

The median LOS was 5 days (IQR: 2-10) days for the COVID period group and 3 days (IQR: 2-6) days for the noCOVID period group $(P<0.001)$. The rate of mortality was slightly higher in the COVID period group (6\% vs. $4.9 \%$ ), but the difference was not significant.

\section{Patients with severe peritonitis}

The subgroup analysis of 202 patients with severe peritonitis-101 in the COVID period group and 101 in the noCOVID period group-revealed no differences in terms of age, BMI, comorbidities, previous abdominal surgery, and the ASA score between the two groups. The COVID period group had a higher proportion of male patients than did the no-COVID period group $(67.3 \%$ vs. $53.5 \% ; P=0.044)$ (Table 3).

Patients treated during the COVID period, in comparison with those treated previously, had longer referral delays [48 h (IQR: $18-72)$ vs. 24 h (IQR: $12-48) ; P=0.004$ ], a higher rate of patients with symptoms onset interval $\geq 48 \mathrm{~h}$ (51.5\% vs. $35.6 \% ; P=0.023)$, higher qSOFA scores $(1-3$ : $24.8 \%$ vs. $12.9 \% ; P=0.031$ ), longer operation times [93 $\mathrm{min}$ (IQR: 60-147) vs. $70 \mathrm{~min}$ (IQR: 50-115); $P=0.005]$, more ileocecectomies for appendicitis $(16.7 \%$ vs. $3 \%$; $P=0.011$ ), higher $\mathrm{CCI} \circledast$ values (75th percentile: 27.9 vs. 8.7; $P=0.048)$, and longer LOS [7 days (IQR: 4-12.5) vs. 5 days (IQR: 3-9.5); $P=0.039$ ]. Rates of postoperative mortality were exactly the same in the two groups $(9 / 101$ patients; 8.9\%) (Tables 3, 4).

Multivariable analysis by complication.

As shown in Table 5, eight potential risk factors were associated with postoperative complications, but only the ASA score $(\mathrm{OR}=3.60 ; 95 \%$ CI $1.82-7.13 ; P<0.001)$, severe peritonitis $(\mathrm{OR}=3.77 ; 95 \%$ CI $1.66-8.52$; $P=0.001)$, diagnosis other than appendicitis $(\mathrm{OR}=2.17$;
Table 2 Operative findings and postoperative outcomes by period of operation

\begin{tabular}{lllr}
\hline Variable & $\begin{array}{l}\text { COVID period } \\
\text { group }(n=149)\end{array}$ & $\begin{array}{l}\text { no-COVID period } \\
\text { group }(n=183)\end{array}$ & $P$ value \\
\hline Severe peritonitis, $n(\%)$ & $101(67.8 \%)$ & $101(55.2 \%)$ & $\mathbf{0 . 0 1 9}$ \\
Laparoscopic approach, $n(\%)$ & $113(75.8 \%)$ & $159(86.9 \%)$ & $\mathbf{0 . 0 0 9}$ \\
Conversion to open, $n(\%)$ & $14(12.4 \%)^{*}$ & $14(8.8 \%)^{*}$ & 0.338 \\
Duration of surgery in minutes, median [IQR] & $75[50-123]$ & $60[40-85]$ & $<\mathbf{0 . 0 0 1}$ \\
Ileocecectomy for appendicitis, $n(\%)$ & $9(9.7 \%)^{* *}$ & $3(2.1 \%)^{* *}$ & $\mathbf{0 . 0 1 3}$ \\
Any complication, $n(\%)$ & $53(35.6 \%)$ & $33(18 \%)$ & $<\mathbf{0 . 0 0 1}$ \\
CCI®, 75th percentile & 20.9 & 0 & $\mathbf{0 . 0 0 1}$ \\
Major complications***, $n(\%)$ & $21(14.1 \%)$ & $16(8.7 \%)$ & 0.123 \\
Death by complication, $n(\%)$ & $9(6 \%)$ & $9(4.9 \%)$ & 0.653 \\
LOS in days, median $[\mathrm{IQR}]$ & $5[2-10]$ & $3[2-6]$ & $<\mathbf{0 . 0 0 1}$ \\
Readmission, $n(\%)$ & $4(2.7 \%)$ & $4(2.2 \%)$ & 0.768 \\
\hline
\end{tabular}

Significant $P$ values are in bold

*Percentages were calculated for the subpopulation of patients undergoing laparoscopy

${ }^{* *}$ Percentages were calculated for the subpopulation of patients undergoing emergency surgery for appendicitis

${ }^{* * *} \geq$ Grade IIIa according to the Clavien-Dindo classification. ${ }^{10}$ 
Table 3 Demographics and preoperative characteristics by period of operation in patients with severe peritonitis

\begin{tabular}{|c|c|c|c|}
\hline Variable & COVID period $(n=101)$ & $\begin{array}{l}\text { no-COVID period } \\
(n=101)\end{array}$ & $P$ value \\
\hline Age in years, median [IQR] & $60[42-75]$ & $54[38.5-73]$ & 0.249 \\
\hline Sex, $n(\%)$ & & & 0.044 \\
\hline Female & $33(32.7 \%)$ & $47(46.5 \%)$ & \\
\hline Male & $68(67.3 \%)$ & $54(53.5 \%)$ & \\
\hline $\mathrm{BMI}$ in $\mathrm{kg} / \mathrm{m}^{2}$, mean $\pm \mathrm{STD}$ & $26.1 \pm 2.3$ & $26 \pm 2.4$ & 0.714 \\
\hline $\mathrm{CACI}>3, n(\%)$ & $29(28.7 \%)$ & $28(27.7 \%)$ & 0.876 \\
\hline Previous abdominal surgery, $n(\%)$ & & & 0.421 \\
\hline No surgery & $73(72.3 \%)$ & $79(78.2 \%)$ & \\
\hline Laparoscopic surgery & $5(5 \%)$ & $2(2 \%)$ & \\
\hline Open surgery & $23(22.8 \%)$ & $20(19.8 \%)$ & \\
\hline WBC count in $10^{9} / \mathrm{L}$, median [IQR] & 14 [11.1-17.9] & $13.4[10-17.3]$ & 0.367 \\
\hline $\mathrm{PMN}$ in $\%$, mean $\pm \mathrm{STD}$ & $82.6 \pm 11.4$ & $82.9 \pm 6.7$ & 0.808 \\
\hline CRP in mg/dL, median [IQR] & $14.9[6.7-30.1]$ & $14[4.9-45.8]$ & 0.910 \\
\hline qSOFA score, $n(\%)$ & & & 0.096 \\
\hline 0 & $74(73.3 \%)$ & $83(82.2 \%)$ & \\
\hline 1 & $19(18.8 \%)$ & $8(7.9 \%)$ & \\
\hline 2 & $6(5.9 \%)$ & $4(4 \%)$ & \\
\hline 3 & 0 & $1(1 \%)$ & \\
\hline Missing & $2(2 \%)$ & $5(4.9 \%)$ & \\
\hline Diagnostic imaging type, $n(\%)$ & & & 0.051 \\
\hline US & $18(17.8 \%)$ & $33(32.7 \%)$ & \\
\hline $\mathrm{CT}$ & $80(79.2 \%)$ & $66(65.3 \%)$ & \\
\hline Plain radiography & $3(3 \%)$ & $2(2 \%)$ & \\
\hline \multicolumn{4}{|l|}{ Imaging findings, $n(\%)$} \\
\hline Abscess & $36(35.6 \%)$ & $40(39.6 \%)$ & 0.561 \\
\hline Free fluid & $71(70.3 \%)$ & $74(73.3 \%)$ & 0.639 \\
\hline Free air & $38(37.4 \%)$ & $34(33.7 \%)$ & 0.557 \\
\hline Referral delay in hours, median [IQR] & $48[21-72]$ & $24[12-48]$ & 0.004 \\
\hline Referral delay $\geq 48$ h, $n(\%)$ & $52(51.5 \%)$ & $36(35.6 \%)$ & 0.023 \\
\hline Operation delay in hours, median [IQR] & $8[5-19.5]$ & $9[4-17]$ & 0.943 \\
\hline Total delay in hours, median [IQR] & $55[21-94]$ & $36[18-72]$ & 0.072 \\
\hline ASA score, $n(\%)$ & & & 0.562 \\
\hline 1 & $25(24.8 \%)$ & $27(26.7 \%)$ & \\
\hline 2 & $36(35.6 \%)$ & $37(36.6 \%)$ & \\
\hline 3 & $27(26.7 \%)$ & $30(29.7 \%)$ & \\
\hline 4 & $13(12.9 \%)$ & $7(6.9 \%)$ & \\
\hline \multicolumn{4}{|l|}{ Cause of peritonitis, $n(\%)$} \\
\hline Perforated peptic ulcer & $17(16.8 \%)$ & $9(8.9 \%)$ & 0.093 \\
\hline Small bowel perforation & $8(7.9 \%)$ & $54(4 \%)$ & 0.234 \\
\hline Appendicitis & $48(47.5 \%)$ & $66(65.3 \%)$ & 0.011 \\
\hline Acute diverticulitis & $28(27.7 \%)$ & $22(21.8 \%)$ & 0.328 \\
\hline
\end{tabular}

Significant $P$ values are in bold
95\%CI: $1.08-4.35 ; P=0.029)$, surgery during the COVID period $(\mathrm{OR}=1.91 ; 95 \% \mathrm{CI} 1.04-3.51 ; P=0.037)$ and qSOFA score $(\mathrm{OR}=1.91 ; 95 \%$ CI $1.01-3.63 ; P=0.049)$ were evidenced as independent predictors according to multivariable analysis.

\section{Discussion}

The results of this study showed that patients undergoing urgent and emergency surgery for secondary peritonitis 
Table 4 Operative findings and postoperative outcomes by period of operation in patients with severe peritonitis

\begin{tabular}{llll}
\hline Variable & $\begin{array}{l}\text { COVID period } \\
\text { group }(n=149)\end{array}$ & $\begin{array}{l}\text { no-COVID period } \\
\text { group }(n=183)\end{array}$ & $P$ value \\
\hline Laparoscopic approach, $n(\%)$ & $69(68.3 \%)$ & $80(79.2 \%)$ & 0.079 \\
Conversion to open, $n(\%)$ & $14(20.3 \%)^{*}$ & $14(17.5 \%)^{*}$ & 0.664 \\
Duration of surgery in minutes, median [IQR] & $93[60-147.5]$ & $70[50-115]$ & $\mathbf{0 . 0 0 5}$ \\
Ileocecectomy for appendicitis, $n(\%)$ & $8(16.7 \%)^{* *}$ & $2(3 \%)^{* *}$ & $\mathbf{0 . 0 1 1}$ \\
Any complication, $n(\%)$ & $45(44.6 \%)$ & $32(31.7 \%)$ & 0.060 \\
CCIß, 75th percentile & 27.9 & 8.7 & $\mathbf{0 . 0 4 8}$ \\
Major complications***, $n(\%)$ & $21(20.8 \%)$ & $15(14.9 \%)$ & 0.270 \\
Death by complication, $\mathrm{n}(\%)$ & $9(8.9 \%)$ & $9(8.9 \%)$ & 1 \\
LOS in days, median $[\mathrm{IQR}]$ & $7[4-12.5]$ & $5[3-9.5]$ & $\mathbf{0 . 0 4 7}$ \\
Readmission, $n(\%)$ & $2(2 \%)$ & $4(4 \%)$ & 0.683 \\
\hline
\end{tabular}

Significant $P$ values are in bold

*Percentages were calculated for the subpopulation of patients undergoing laparoscopy

** Percentages were calculated for the subpopulation of patients undergoing emergency surgery for appendicitis

${ }^{* *} \geq$ Grade IIIa according to the Clavien-Dindo classification. ${ }^{10}$

\begin{tabular}{|c|c|c|c|c|c|}
\hline \multirow[t]{2}{*}{ Variable } & \multicolumn{3}{|c|}{ Univariate analysis } & \multicolumn{2}{|c|}{ Multivariate analysis } \\
\hline & $\begin{array}{l}\text { Complicated } \\
\text { patients } \\
(n=86)\end{array}$ & $\begin{array}{l}\text { Uncomplicated } \\
\text { patients }(n=246)\end{array}$ & $P$ value & OR $[95 \% \mathrm{CI}]$ & $P$ value \\
\hline Age in years, median [IQR] & 64.5 [46.5-79] & $39[22-57]$ nnnnn & $<0.001$ & $0.99[0.97-1.01]$ & 0.358 \\
\hline Sex, $n(\%)$ & & & 0.378 & & \\
\hline Male & $46(53.5 \%)$ & $145(58.9 \%)$ & & & \\
\hline Female & $40(46.5 \%)$ & $101(41.1 \%)$ & & & \\
\hline $\mathrm{BMI}$ in $\mathrm{kg} / \mathrm{m}^{2}$, mean $\pm \mathrm{STD}$ & $26.8 \pm 2.5$ & $25.9 \pm 2.3$ & 0.179 & & \\
\hline $\mathrm{CACI}>3, n(\%)$ & $36(41.9 \%)$ & $29(11.8 \%)$ & $<0.001$ & $1.69[0.68-4.22]$ & 0.260 \\
\hline Period, $n(\%)$ & & & $<0.001$ & $1.91[1.04-3.51]$ & $\mathbf{0 . 0 3 7}$ \\
\hline COVID period & $53(61.6 \%)$ & $96(39 \%)$ & & & \\
\hline No-COVID period & $33(38.4 \%)$ & $150(61 \%)$ & & & \\
\hline qSOFA score, $n(\%)$ & & & $<0.001$ & $1.91[1.01-3.63]$ & 0.049 \\
\hline 0 & $59(68.6 \%)$ & $225(94.1 \%)$ & & & \\
\hline 1 & $16(18.6 \%)$ & $13(5.4 \%)$ & & & \\
\hline 2 & $10(11.6 \%)$ & $1(0.4 \%)$ & & & \\
\hline 3 & $1(1.2 \%)$ & 0 & & & \\
\hline Missing & 0 & $7(2.8 \%)$ & & & \\
\hline Referral delay $\geq 48$ h, $n(\%)$ & $44(51.2 \%)$ & $77(31.3 \%)$ & 0.001 & $1.62[0.88-3]$ & 0.125 \\
\hline $\begin{array}{l}\text { Diagnosis other than appen- } \\
\text { dicitis, } n(\%)\end{array}$ & $53(61.6 \%)$ & $41(16.7 \%)$ & $<0.001$ & $2.17[1.08-4.35]$ & 0.029 \\
\hline ASA score, $n(\%)$ & & & $<0.001$ & $3.60[1.82-7.13]$ & $<0.001$ \\
\hline 1 & $11(12,8 \%)$ & $122(49,6 \%)$ & & & \\
\hline 2 & $22(25.6 \%)$ & $91(37 \%)$ & & & \\
\hline 3 & $35(40.7 \%)$ & $31(12.6 \%)$ & & & \\
\hline 4 & $18(20.9 \%)$ & $2(0.8 \%)$ & & & \\
\hline Severe peritonitis, $n(\%)$ & & & $<0.001$ & $3.77[1.66-8.52]$ & 0.001 \\
\hline Yes & $77(89.5 \%)$ & $125(50.8 \%)$ & & & \\
\hline No & $9(10.5 \%)$ & $121(49.2 \%)$ & & & \\
\hline
\end{tabular}

Significant $P$ values are in bold
Table 5 Univariate and multivariate analysis by complication 
during the COVID-19 period experienced more postoperative complications than did patients treated the year before.

The ongoing pandemic, both in its first and second surge, is putting physicians, healthcare providers and health systems under stress, and not only because of the dramatic effects of COVID-19 on infected individuals. As the contagion spreads, we find ourselves in a global crisis characterized by a continuously mutating scenario, where the word "unprecedented" has taken on a new meaning. The COVID19 pandemic advocated a constrained reshaping of healthcare services in Italy, which changed from region to region according to the policies of autonomous local healthcare administrations. The high rate of hospitalization of patients with COVID-19 has forced the creation of differentiated routes of access to EDs [17], especially in areas where the infection is highly prevalent. Regarding surgery practice, after the implementation of viral screening tests with rapid polymerase chain reaction, Emilia-Romagna region drafted a protocol to identify infected individuals among patients requiring unscheduled surgery, creating differentiated wards for infected patients, noninfected patients, and patients waiting for virological test results. Specifically, candidates for urgent surgical treatment underwent oropharyngeal swab tests for rapid SARS-CoV-2 research upon their arrival in the ED and were brought to the operating room once the result was available (approximately four hours later). In contrast, unstable patients were brought to the operating room and managed as if they were infected on a precautionary basis; test samples were obtained by oropharyngeal swab or bronchoalveolar lavage during anesthesia induction so that test results were available at the end of the intervention [18]. In response to the many concerns raised about the possibility of laparoscopy causing aerosolization of viral particles $[19,20]$, all laparoscopic procedures were conducted with systems of smoke evacuation and gas filtration.

Since the beginning of the epidemic in China, COVID-19 has received broad media coverage in Italy, and this coverage became massive after the first case was identified in Lombardy region. As a result of the narrow focus on the pandemic by media and the increasing numbers of infected individuals and COVID-19-related deaths, many members of the general population began to avoid seeking medical care out of fear of infection, even before lockdown measures were implemented. The fear of infection has dramatically changed the perception of hospitals among the general population [4]; fewer people have sought healthcare services, similar to behavior described during the 2004 SARS outbreak in Taiwan and the 2014 Ebola outbreak in Africa [21, 22]. The observation of Italian general surgeons is that throughout the outbreak, patients have sought medical attention belatedly, because of the fear of exposure to SARS-CoV-2 inside the hospital. This observation not only led us to conceive and design this study but also has raised a deep concern: how can we help our patients when they themselves are afraid to seek care?

The first obvious difference between the times before and during the pandemic is the increased delay in referral during COVID-19 period, which may support the assumptions about fear-induced hospital avoidance. The difference in median referral delay between the two periods is only four hours, and-although statistically significant—-this finding may not imply a clinical significancy. However, it is evident that referral delays during the COVID-19 period are characterized by a larger statistical dispersion with higher deviations from the median (Fig. 1), especially in the last quartiles. Also, patients operated during that period, have shown a higher rate of referral delays $\geq 48 \mathrm{~h}$ ( $43 \%$ vs. $31.1 \%$ ). The reasons that may justify such delay remain mostly speculative and could not be measured in this study, although many recent reports have highlighted people's concern over risking infection during medical care $[4,5]$. Nevertheless, the total interval of time between symptoms onset and surgical intervention in the two periods has been comparable, suggesting that operation delay might have counterbalanced referral delay, although no significant differences have been evidenced. These findings strengthen the assumption that throughout the COVID-19 outbreak the willingness to seek medical aid has been the weak link in the healthcare process. In this sense, the increased frequency of abdominal free fluid at preoperative imaging in the COVID period group may imply that the duration of the peritoneal inflammation process was longer, although relevant data in the medical literature are inconsistent. Of interest is that during the lockdown period, diagnosis was accomplished mostly through CT. To a first assumption, this may be explained by the worse clinical conditions of the patients, also reflected by the elevated qSOFA scores in the COVID period group; however throughout the COVID-19 outbreak, every potential cause of fever was included in the differential diagnosis, along with SARS-CoV-2 infection, which was more likely to be detected through CT.

Severe peritonitis, according to AAST classifications, was relatively more frequent among patients in the COVID period group. However, the number of patients with severe peritonitis was exactly the same in the two groups (101 patients each). Therefore, the incidence of severe peritonitis may not have increased; rather, the proportion of cases of mild peritonitis found during intraoperative exploration was lower. In this sense, the imbalance in diagnoses between the two periods plays a relevant role: in fact during the COVID period the number of patients referred to surgery for appendicitis was lower both in proportion and absolute number, with a higher frequency of peritonitis secondary to other causes (acute diverticulitis, small bowel perforation or perforated peptic ulcer). The reduced frequency of appendicitis 
referred to surgery during the COVID period has also been evidenced in a multicentric retrospective study based in Israel by Tankel et al. [23] Although the reasons behind this evidence could not be investigated in the setting of this study, we agree that this finding is likely related to successful resolution of mild appendicitis treated by patients or general practitioners at home, and to a general shift towards nonoperative management of mild peritonitis, as suggested by international recommendations published online during the pandemic [24].

Not only peritonitis appeared to be less frequently mild throughout the COVID-19 outbreak, but it also produced stronger systemic effects, as evidenced by the worse qSOFA scores of the COVID-19 period cohort. The increased numbers of patients with signs of sepsis-related organ dysfunction together with slight etiological differences may also justify the reduced use of laparoscopy. However, in the two cohorts the rates of conversion to open procedures were comparable, which suggests that the appropriateness of the surgical indications was maintained. As expected, because more severe peritonitis was found in surgical exploration during the COVID-19 period, the duration of surgical procedures increased.

Although patients presented to the ED with comparable individual profiles of preoperative risk, the cohort of patients who underwent surgery during the COVID-19 lockdown exhibited an almost doubled rate of surgical complications ( $35.6 \%$ vs. $18 \%$ ), with increased CCI ${ }^{\circledR}$ values. This increased rate of postoperative complications is both evident in the subgroup of patients with mild peritonitis $(8 / 40$, $16.7 \%$ vs. $1 / 81,1.2 \%, P=0.001)$ and in the subgroup of patients with severe peritonitis, although in the latter such difference does not reach a statistical significancy $(44.6 \%$ vs. $31.7 \% ; \mathrm{P}=0.060$ ). In support of this suggestion, the length of hospitalization was significantly increased during the COVID-19 period. These findings illustrate a critical scenario: throughout a period in which healthcare resources and funds have been concentrated on the pandemic, an increased burden of surgical complications has increased both hospital costs and assistance efforts.

The patients who underwent surgery during the COVID19 period had a lower incidence of appendicitis, a reduced rate of mild peritonitis, a higher rate of delayed presentations to the EDs and poorer qSOFA scores compared to those operated during the same period of year 2019. Most of those phenomena appeared to be strong predictors of postoperative complications, and their cumulative effect may explain why the COVID-19 period itself resulted a predictor of complications. The reasons behind the sharp shift in terms of diagnoses and severity of peritonitis which was observed are, in the setting of this study, mostly speculative. Nevertheless, both the imbalance of diagnoses and the rate of delayed presentations to the EDs may imply that fear of contagion drove patients to avoid in-hospital care: in this sense it is possible to hypothesize that patients who experienced abdominal symptoms only presented to hospitals when symptoms overwhelmed fear or when domiciliary therapy failed. This assumption may suggest that, as many authors reported [6-9, 25-28], the pandemic has dramatically influenced the delivery of ordinary healthcare services in many fields.

Not least, during the COVID pandemic healthcare providers have been pressured with high psychological tension derived from adjunctive shifts in COVID wards, reduction in staff as a result of SARS-CoV-2 infection, burnout, personal stress, worry about infecting the family members and forced isolation [29]. Although those aspects are far from the framing capacity of this experimental setting, it is not illegitimate to hypothesize a role of psychological stress on the outcomes of patients who received non-COVID related care during the pandemic.

This study has some limitations. First, its prospective case-retrospective cohort design affected our ability to draw definite conclusions; nevertheless, given the extraordinary circumstances in which this study was conducted, this kind of design was the most reliable and affordable option. Also, the limited number of patients may have influenced the results. Another limitation of the study was the impossibility of testing all patients for SARS-CoV-2 infection during the first part of the COVID-19 period to exclude with certainty patients with preoperative infection, since the screening protocol was implemented a few days after the beginning of the study; in this short period, patients were screened with high-resolution chest $\mathrm{CT}$ and evaluation of symptoms. Finally, the lack of data regarding the population of patients who underwent nonoperative management (especially for what concerns cases of appendicitis) has circumscribed the focus of the study.

\section{Conclusions}

Undoubtedly, the COVID-19 outbreak has had a drastic effect on healthcare providers and is a previously unimaginable "once-in-a-generation" worldwide emergency. Although the tactical sanitary response to the incoming disease was drafted weeks before the first cases in Europe were identified, many potential interferences of the epidemic with ordinary medical and surgical emergencies were underestimated. For what concerns emergency and urgent surgical activity, the COVID period has been characterized with a reduction in mild peritonitis cases, likely related to a shift towards non operative management and domiciliary treatment. As a result, an increased rate of patients with higher chances of postoperative complications weighed on hospital expenses and healthcare providers during a period of extreme effort for sanitary assistance. 
Acknowledgements The Authors would like to thank Dr. Andrea Romboli, MD and Dr. Gerti Dajti, MD for their help in the idea of the research.

Funding The authors have no sources of funding/support to declare.

\section{Compliance with ethical standards}

Conflict of interest The authors have non conflicts of interest to disclose.

Ethics approval This study was reviewed and approved by the Ethics Committee.

Informed consent Informed consent for enrollment was obtained from all individual participants involved in the study.

\section{References}

1. Grasselli G, Pesenti A, Cecconi M (2020) Critical care utilization for the COVID-19 outbreak in Lombardy, Italy: early experience and forecast during an emergency response. JAMA. https://doi. org/10.1001/jama.2020.4031 ((Epub ahead of print))

2. European Centre for Disease Prevention and Control. COVID-19 situation update for the EU/EEA and the UK. https://www.ecdc. europa.eu/en/cases-2019-ncov-eueea. Accessed 5 Nov 2020

3. Health Ministry's website. COVID-19 Italy - Daily situation report. http://www.salute.gov.it/portale/nuovocoronaviru s/dettaglioContenutiNuovoCoronavirus.jsp?lingua=engli sh\&id $=5367 \&$ area $=$ nuovoCoronavirus $\&$ menu $=$ vuoto. Accessed 5 Nov 2020

4. Saad L. Americans worry doctor visits raise COVID-19 risk. April 6, 2020. https://news.gallup.com/poll/307640/americans-worry -doctor-visits-raise-covid-risk.aspx. Accessed 20 Apr 2020

5. Mantica G, Riccardi N, Terrone C, Gratarola A (2020) NonCOVID-19 visits to emergency departments during the pandemic: the impact of fear. Public Health 183:40-41

6. De Rosa S, Spaccarotella C, Basso C, Calabrò MP, Curcio A, Perrone Filardi P et al (2020) Reduction of hospitalizations for myocardial infarction in Italy in the COVID-19 era. Eur Heart J 41:2083-2088

7. Lazzerini M, Barbi E, Apicella A, Marchetti F, Cardinale F, Trobia G (2020) Delayed access or provision of care in Italy resulting from fear of COVID-19. Lancet Child Adolesc Health 4:e10-e11

8. Hoyer C, Ebert A, Szabo K, Platten M, Meyer-Lindenberg A, Kranaster L (2020) Decreased utilization of mental health emergency service during the COVID-19 pandemic. Eur Arch Psychiatry Clin Neurosci. https://doi.org/10.1007/s00406-020-01151-w ((Epub ahead of print))

9. Snapiri O, Rosenberg Danziger C, Krause I et al (2020) Delayed diagnosis of pediatric appendicitis during the COVID-19 pandemic. Acta Paediatr 109:1672-1676

10. Dindo D, Demartines N, Clavien PA (2004) Classification of surgical complications: a new proposal with evaluation in a cohort of 6336 patients and results of a survey. Ann Surg 240:205-213

11. Slankamenac K, Graf R, Barkun J, Puhan MA, Clavien PA (2013) The comprehensive complication index: a novel continuous scale to measure surgical morbidity. Ann Surg 258:1-7

12. Seymour CW, Liu VX, Iwashyna TJ, Brunkhorst FM, Rea TD, Scherag A et al (2016) Assessment of clinical criteria for sepsis: for the third international consensus definitions for sepsis and septic shock (Sepsis-3). JAMA 315:762-774
13. Shafi S, Aboutanos M, Brown CV, Ciesla D, Cohen MJ, Crandall ML et al (2014) Measuring anatomic severity of disease in emergency general surgery. J Trauma Acute Care Surg 76:884-887

14. Charlson M, Szatrowski TP, Peterson J, Gold J (1994) Validation of a combined comorbidity index. J Clin Epidemiol 47:1245-1251

15. St-Louis E, Iqbal S, Feldman LS, Sudarshan M, Deckelbaum DL, Razek TS et al (2015) Using the age-adjusted Charlson comorbidity index to predict outcomes in emergency general surgery. $\mathrm{J}$ Trauma Acute Care Surg 78:318-323

16. American Society of Anesthesiologists (1963) A new classification of physical status. Anesthesiology 24:111

17. Garcia-Castrillo L, Petrino R, Leach R, Dodt C, Behringer W, Khoury A et al (2020) European Society For Emergency Medicine position paper on emergency medical systems' response to COVID-19. Eur J Emerg Med 27:174-177

18. De Simone B, Chouillard E, Di Saverio S, Pagani L, Sartelli M, Biffl WL et al (2020) Emergency surgery during the COVID-19 pandemic: what you need to know for practice. Ann R Coll Surg Engl 102:323-332

19. Zheng MH, Boni L, Fingerhut A (2020) Minimally invasive surgery and the novel coronavirus outbreak: lessons learned in China and Italy. Ann Surg 272:e5-e6

20. Francis N, Dort J, Cho E, Feldam L, Keller D, Lim R et al (2020) SAGES and EAES recommendations for minimally invasive surgery during COVID-19 pandemic. Surg Endosc 34:2327-2331

21. Chang HJ, Huang N, Lee CH, Hsu Y, Hsieh C, Chou Y (2004) The impact of the SARS epidemic on the utilization of medical services: SARS and the fear of SARS. Am J Public Health 94:562-564

22. Bali S, Stewart KA, Pate MA (2016) Long shadow of fear in an epidemic: fearonomic effects of Ebola on the private sector in Nigeria. BMJ Glob Health 1:e000111

23. Tankel J, Keinan A, Blich O, Koussa M, Helou B, Shay S et al (2020) The decreasing incidence of acute appendicitis during COVID-19: a retrospective multi-centre study. World J Surg $44: 2458-2463$

24. Moletta L (2020) International guidelines and recommendations for surgery during Covid-19 pandemic: a systematic review. Int J Surg 79:180-188

25. Rosenbaum L (2020) The untold toll — the pandemic's effects on patients without Covid-19. N Engl J Med 382:2368-2371

26. Kansagra AP, Goyal MS, Hamilton S, Albers GW (2020) Collateral effect of Covid-19 on stroke evaluation in the United States. N Engl J Med 383:400-401

27. Haut ER, Leeds I, Livingston DH (2020) The effect on trauma care secondary to the COVID-19 pandemic: collateral damage from diversion of resources. Ann Surg. https://doi.org/10.1097/ SLA0000000000004105 ((Epub ahead of print))

28. Negrini S, Grabljevec K, Boldrini P, Kiekens C, Moslavac S, Zampolini $\mathrm{M}$ et al (2020) Up to 2.2 million people experiencing disability suffer collateral damage each day of Covid-19 lockdown in Europe. Eur J Phys Rehabil Med 56:361-365

29. El-Hage W, Hingray C, Lemogne C, Yrondi A, Brunault P, Bienvenu $\mathrm{T}$ et al (2020) Les professionnels de santé face à la pandémie de la maladie à coronavirus (COVID-19): quels risques pour leur santé mentale ? [Health professionals facing the coronavirus disease 2019 (COVID-19) pandemic: What are the mental health risks?]. Encephale 46:S73-S80

Publisher's Note Springer Nature remains neutral with regard to jurisdictional claims in published maps and institutional affiliations. 